\title{
Schrifttum zum Studium des Strafrechts, Allgemeiner Teil
}

Der Bezug auf den GrundKurs Strafrecht, B.T., betrifft den GrundKurs STRAFreChT, Die einzelnen Delikte, 4. Aufl. 1995.

\section{I. Älteres Schrifttum}

1. Lehrbücher und Grundrisse

BINDING

BINDING

BINDING

MAYER, M.E. V. HIPPEL, ROBERT

BELING

v. LISZT/SCHMIDT

GeRLAND

MEZGeR

2. Kommentare

FRANK, R.

v. Olshausen
Handbuch des Strafrechts, Bd. I, 1885

Grundriß des Deutschen Strafrechts, Allgemeiner Teil, 8. Aufl. 1913, Nachdruck 1975 Die Normen und ihre Übertretung, Bd. I (4. Aufl. 1922), Bd. II (2. Aufl. 1914 - 1916), Bd. III (1918), Bd. IV (1919)

Der Allgemeine Teil des deutschen Strafrechts, 2. Aufl. 1923 Deutsches Strafrecht, Bd. I (1925), Bd. II (1930)

Grundzüge des Strafrechts, 11. Aufl. 1930

Lehrbuch des deutschen Strafrechts, Bd. I, Allgemeiner Teil, 26. Aufl. 1932

Deutsches Reichsstrafrecht, 2. Aufl. 1932

Strafrecht, Ein Lehrbuch, 3. (unv.) Aufl. 1949

Das Strafgesetzbuch für das Deutsche Reich, 18. Aufl. 1931 mit Nachtrag 1936 (Schäfer/v. Dohnanyi)

Kommentar zum Strafgesetzbuch für das Deutsche Reich, Bd. I, 12. Aufl. 1942/1943

\section{Neueres Schrifttum}

1. Einführung in das Strafrecht

\section{HASSEMER}

NAUCKE

ROXIN/ARzT/

TIEDEMANN

SCHMIDHÄUSER
Einführung in die Grundlagen des Strafrechts, 2. Aufl. 1990

Strafrecht, Eine Einführung, 7. Aufl. 1995

Einführung in das Strafrecht und Strafprozeßrecht, 3. Aufl. 1994

Einführung in das Strafrecht, 2. Aufl. 1984

2. Lehrbücher und Grundrisse

BAUMANN/WEBER/

MITSCH

BLEI

BOCKELMANN/VOLK

EBERT

ESER/BURKHARDT
Strafrecht, Allgemeiner Teil, 10. Aufl. 1995

Strafrecht I, Allgemeiner Teil, 18. Aufl. 1983

Strafrecht, Allgemeiner Teil, 4. Aufl. 1987

Strafrecht, Allgemeiner Teil, 2. Aufl., 1994

Strafrecht, Bd. 1: Allgemeine Verbrechenselemente, 4. Aufl. 1992 
ESER

HAFT

HAUF

HRUSCHKA

JAKOBS

JESCHECK/WEIGAND

KIENAPFEL

KÜHL

MAURACH/ZIPF

MAURACH/GÖSSEL/ZIPF

MAYER, $\mathrm{H}$.

MAYER, $\mathrm{H}$.

ROXIN

\section{SAUER}

SCHMIDHÄUSER

SCHMIDHÄUSER

STRATENWERTH

WELZEL

WESSELS

\section{Kommentare}

DREHER/TRÖNDLE

KOHLRAUSCH/LANGE

LACKNER

LEIPZIGER

KOMMENTAR (LK)

Nomos KoMmEnTAR

Pfeiffer/Maul/

SCHUlte

PREISENDANZ

SCHÖNKE/SCHRÖDER

SYSTEMATISCHER

KOMMENTAR (SK)
STRAFRECHT Bd. 2: Fahrlässigkeit, Unterlassung, Versuch, Teilnahme, 3. Aufl. 1980

Strafrecht, Allgemeiner Teil, 6. Aufl. 1994

Strafrecht, Allgemeiner Teil, 1996

Strafrecht nach logisch-analytischer Methode, 2. Aufl. 1988

Strafrecht, Allgemeiner Teil, 2. Aufl. 1991

Lehrbuch des Strafrechts, Allgemeiner Teil, 5. Aufl. 1996

Strafrecht, Allgemeiner Teil, 5. Aufl. 1994

Strafrecht, Allgemeiner Teil, 1994

Strafrecht, Allgemeiner Teil, Tbd. 1, 8. Aufl. 1992

Strafrecht, Allgemeiner Teil, Tbd. 2, 7. Aufl. 1989

Das Deutsche Strafrecht, 2. Aufl., 1953

Strafrecht, Allgemeiner Teil, 1967

Strafrecht, Allgemeiner Teil, Bd. I, Grundlagen,

Aufbau der Verbrechenslehre, 2. Aufl., 1994

Allgemeine Strafrechtslehre, 3. Aufl. 1955

Strafrecht, Allgemeiner Teil, 2. Aufl. 1975

Strafrecht, Allgemeiner Teil, Studienbuch, 2. Aufl. 1984

Strafrecht, Allgemeiner Teil I, Die Straftat, 3. Aufl. 1981

Das deutsche Strafrecht, 11. Aufl. 1969

Strafrecht, Allgemeiner Teil, 25. Aufl. 1995

Strafgesetzbuch und Nebengesetze, 47. Aufl. 1995

Strafgesetzbuch mit Erläuterungen und Nebengesetzen, 43.

Aufl. 1961

Strafgesetzbuch mit Erläuterungen, 21. Aufl. 1995

Großkommentar zum Strafgesetzbuch,

10. Aufl. ,1978 ff., hrsg. v. Jescheck, Ruß und Willms,

11. Aufl., 1992 ff., hrsg. v. Jähnke, Laufhütte und Odersky

zum Strafgesetzbuch, hrsg. v. Neumann und Schield, 1995 ff, Stand: April 1996.

Strafgesetzbuch, Kommentar an Hand der Rechtsprechung des Bundesgerichtshofes, 1969

Strafgesetzbuch, Lehrkommentar, 30. Aufl. 1978

Strafgesetzbuch, bearb. von Cramer, Eser, Lenckner und Stree, 24. Aufl. 1991

zum Strafgesetzbuch, bearb. von Rudolphi, Horn, Günther, Samson, Allgemeiner Teil, 6. Aufl. , Stand August 1995

4. Kommentare zu strafrechtlichen Nebengesetzen

DALCKE/FUHRMANN/

SCHÄFER

ERBS/KOHLHAAS
Strafrecht und Strafverfahren, 37. Aufl. 1961

Strafrechtliche Nebengesetze (Loseblattsammlung) 
Schrifttum

\section{Strafrechtswissenschaftliche Zeitschriften}

Goltdammer's Archiv für Strafrecht (GA)

Monatsschrift für Kriminologie und Strafrechtsreform (MschrKrim)

Neue Zeitschrift für Strafrecht (NStZ)

Strafverteidiger (StV)

Zeitschrift für Wirtschaft, Steuer, Strafrecht (wistra)

Zeitschrift für die gesamte Strafrechtswissenschaft (ZStW)

\section{Verzeichnis der im Text angeführten Festschriften/Gedächtnisschriften}

BAUMANN, JÜRGEN

BENGL, KARL

BLAU, GÜNTER

BOCKELMANN, PAUL

BRUNS, HANS-JÜRGEN

BUNDESGERICHTSHOF

CoING, Helmut

DEUTSCHER

JURISTENTAG

DREHER, EDUARD

DÜNNEBIER, HANNS

ENGISCH, KARL

GAGNER, STEN

GALLAS, WILHELM

GEERDS, FRIEDRICH

V. GLEISPACH, WENZEL

GolTDAMMER's ARCHIV

GRÜNHUT, MAX

HEINITZ, ERNST

HENKEL, HEINRICH

HONIG, RiCHARD M.

JESCHECK, HANS-HEINR.

KaUfManN, ARMIN

KAUFMANN, HiLdE

KERN, EDUARD

KLEINKNECHT, TH.

KLUG, ULRICH

KOHLRAUSCH, ED.

KRAUSE, FRIEDRICH W.

LACKNER, KARL

LANGE, HEINRICH
Festschrift zum 70. Geburtstag, 1992, Bielefeld

Festschrift, 1984, München

Festschrift zum 70. Geburtstag, 1985, Berlin

Festschrift zum 70. Geburtstag, 1979, München

Festschrift zum 70. Geburtstag, 1978, Köln

Festschrift zum 25jährigen Bestehen, "25 Jahre Bundesgerichtshof", 1975, München

Festschrift zum 70. Geburtstag, "Europäisches Rechtsdenken in Geschichte und Gegenwart", 1982, München

Festschrift zum 100jährigen Bestehen, "Hundert Jahre

Deutsches Rechtsleben", 1960, Karlsruhe

Festschrift zum 70. Geburtstag, 1977, Berlin

Festschrift zum 75. Geburtstag, 1982, Berlin

Festschrift zum 70. Geburtstag, 1969, Frankfurt

Festschrift zum 70. Geburtstag, 1991, München

Festschrift zum 70. Geburtstag, 1973, Berlin

Festschrift zum 70. Geburtstag, 1995, Lübeck

Festschrift zum 60. Geburtstag, "Gegenwartsfragen der

Strafrechtswissenschaft", 1936, Berlin

Festschrift zum 140-jährigen Bestehen, 1993, Heidelberg

Erinnerungsgabe, 1965, Marburg

Festschrift zum 70. Geburtstag, 1972, Berlin

Festschrift zum 70. Geburtstag, "Grundfragen der gesamten

Strafrechtswissenschaft", 1974, Berlin

Festschrift zum 80. Geburtstag, 1970, Göttingen

Festschrift zum 70. Geburtstag, 1985, Berlin

Gedächtnisschrift, 1989, Köln

Gedächtnisschrift, 1986, Berlin

Tübinger Festschrift zum 80. Geburtstag, 1968, Tübingen

Festschrift zum 75. Geburtstag, "Strafverfahren im

Rechtsstaat", 1985, München

Festschrift zum 70. Geburtstag, 1983, Köln

Festschrift zum 70. Geburtstag, "Probleme der

Strafrechtserneuerung", 1944, Berlin

Festschrift zum 70. Geburtstag, 1990, Köln

Festschrift zum 70. Geburtstag, 1987, Berlin

Festschrift zum 70. Geburtstag, 1990, München 
LANGE, HermanN

LANGE, RICHARD

LEFERENZ, HEINZ

MaRTENS, WolfGang

MAURACH, REINHART

MAYER, HELLMUTH

MESSNER, JOHANNES

MeYer, KaRLheinZ

MEZGER, EDMUND

MiYazawa, KoICHI

NOLL, PETER

OBERLANDESGERICHT

Celle

OEHLER, Dietrich

PETERS, KARL

Pfeiffer, Gerd

REBMANN, KuRT

RITTLER, THEODOR

SALGER, HANNSKARL

SARSTEDT, WERNER

SCHAFFSTEIN, FRIEDR.

SCHMIDT, EBERHARD

SCHRÖDER, HORST

SCHÜLER-

SPRINGORUM, HoRST

SCHWINGE, ERICh

v. SIMSON, WERNER

SPANN, WOLFGANG

SPENDEL, GÜNTER

STOCK, ULRICH

Stree, Walter/

WESSELS, JOHANNES

TRIFFTERER, OTTO

TRÖNDLE, HERBERT

UNIVERSITÄT

HEIDELBERG

WALTER, ROBERT
Festschrift zum 70. Geburtstag, 1992, Stuttgart

Festschrift zum 70. Geburtstag, 1976, Berlin

Festschrift zum 70. Geburtstag, "KriminologiePsychiatrie-Strafrecht", 1983, Heidelberg

Gedächtnisschrift, 1987, Berlin

Festschrift zum 70. Geburtstag, 1972, Karlsruhe

Festschrift zum 70. Geburtstag, "Beiträge zur gesamten Strafrechtswissenschaft", 1966, Berlin

Festschrift zum 85. Geburtstag, "Ordnung im sozialen Wandel", 1976, Berlin

Gedächtnisschrift, 1990, Berlin

Festschrift zum 70. Geburtstag, 1954, München

Festschrift, 1995, Baden-Baden

Gedächtnisschrift, 1984, Zürich

Göttinger Festschrift zum 250-jährigen Bestehen, 1961,

Göttingen

Festschrift zum 70 Geburtstag, 1985, Köln

Festschrift zum 70. Geburtstag, "Einheit und Vielfalt des Strafrecht, 1974, Tübingen

Festschrift zum Abschied aus dem Amt als Präsident des Bundesgerichtshofes, "Strafrecht, Unternehmensrecht, Anwaltsrecht", 1988, Köln

Festschrift zum 65. Geburtstag, 1989, München

Festschrift zum 80. Geburtstag, 1957, Aalen

Festschrift zum Abschied aus dem Amt als Vizepräsident des BGH, 1995, Köln, München, u.a.

Festschrift zum 70. Geburtstag, 1981, Berlin

Festschrift zum 70. Geburtstag, 1975, Göttingen

Festschrift zum 70. Geburtstag, 1961, Göttingen

Gedächtnisschrift, 1978, München

Festschrift zum 65. Geburtstag, 1993, Köln, Berlin, Bonn, München

Festschrift zum 70. Geburtstag, "Persönlichkeit in der Demokratie", 1973, Köln

Festschrift zum 75. Geburtstag, 1983, Baden-Baden

Festschrift zum 65. Geburtstag, "Medizin und Recht", 1986, Berlin

Festschrift zum 70. Geburtstag, 1992, Berlin

Festschrift zum 70. Geburtstag, "Studien zur Strafrechtswissenschaft", 1966, Würzburg

Festschrift zum 70. Geburtstag, 1993, Heidelberg

Festschrift zum 65. Geburtstag, 1996, Wien u.a.

Festschrift zum 70. Geburtstag, 1989, Berlin

Festschrift der Juristischen Fakultät zur 600-Jahr-Feier,

"Richterliche Rechtsfortbildung", 1986, Heidelberg

Festschrift zum 60. Geburtstag, 1991, Wien 
WASSERMANN, R.

v. WEBER, HELMUTH

WEIBAUER, WALTHER

WELZEL, HANS

WOLF, ERNST

WÜRTENBERGER,

THOMAS
Festschrift zum 60. Geburtstag, 1985, Darmstadt

Festschrift zum 70. Geburtstag, 1963, Bonn

Festschrift zum 65. Geburtstag, "Ärztliches Handeln Verrechtlichung eines Berufsstandes", 1986, Berlin

Festschrift zum 70. Geburtstag, 1974, Berlin

Festschrift zum 70. Geburtstag, "Recht und Rechtserkenntnis", 1985, Köln

Festschrift zum 70. Geburtstag, "Kultur-Kriminalität-

Strafrecht", 1977, Berlin 
